\title{
EVALUACIÓN DE L.A EFECTIVIDAD ANESTÉSICA DE L.A ACUPUNTURA COMPARADA CON LA LIDOCAÍNA EN PROCEDIMIENTOS ODONTOLÓGICOS DE OPERATORIA: ESTUDIO PRELIMINAR
}

'Diana Patricia González E., ${ }^{2}$ Raúl Blanco G.

'Estudiante de X semestre, Facultad de Odontologia, U. Santo Tomás

${ }^{2}$ Médico - cirujano, Especialista en Medicina Tradicional China, Docente U. Santo Tomás

Autor responsable de correspondencia: Diana Patricia González E.

Correo electrónico:d_gonzalez@medscape.com

\begin{abstract}
RESUMEN
Objetivo: Evaluar la efectividad de la acupuntura comparada con la lidocaina para producir anestesia durante los procedimientos odontológicos de operatoria.

Materiales y métodos: Se desarrolló un estudio experimental de tipo ensayo clínico no controlado en 41 individuos de 18 a 45 años, asistentes a las clínicas odontológicas de la Universidad Santo Tomás, que requerian tratamientos de operatoria. Se estableció la incidencia de dolor en los dos grupos de tratamiento y se describieron los posibles efectos secundarios en las dos intervenciones.

Resultados: En total se evaluaron 41 individuos, el 36.6\% pertenecian al grupo de acupuntura ( $\mathrm{n}=15$ ), y el $63.4 \%$, al grupo de lidocaina $(\mathrm{n}=26)$. La distribución por género mostró para mujeres $58.54 \%$, y para hombres $41.46 \%$, con un promedio de edad de 25.7 y 31.15 años. De los 41 individuos evaluados, 16 (39.02\%) sintieron dolor durante el tratamiento, 16 (39.02\%) tuvieron adormecimiento muscular; 13 (31.71\%) sintieron dolor al insertar la aguja en el tejido; 8 (19.51\%) presentaron sangrado o hematoma; 4 (9.76\%) presentaron irritación; 3 (7.32\%) registraron dolor de cabeza, y 1 (2.44\%) mostró sudoración. Conclusiones: Se podría sugerir que ambas técnicas son iguales en efectividad para los procedimientos de operatoria simple. Por medio de la acupuntura se puede evitar $(60 \%)$ o disminuir (40\%) el dolor ocasionado durante los procedimientos operatorios, para eliminar la dosis del fármaco administrado con este fin. Los resultados observados y la preferencia de los individuos que participaron, permiten concluir que se pueden obtener buenos resultados si la técnica es adecuada. [González D, Blanco R. Evaluación de la efectividad anestésica de la acupuntura comparada con la lidocaina en procedimientos odontológicos de operatoria: Estudio preliminar. Ustasalud Odontología 2004; 3: 86 - 91]
\end{abstract}

Palabras clave: Acupuntura, Lidocaina, Dolor dental, Anestesia.

\section{EVALUATION OF THE ANAESTHETIC EFFECTIVENESS OF ACUPUNCTURE AND LYDOCAINE IN RESTORATIVE DENTAL. PROCEDURES: A PRELIMINARY REPORT}

\begin{abstract}
Objective: To assess the effectiveness of the acupuncture compared with the lydocaine to produce anesthesia during dental restorative procedures.

Materials and methods: A not controlled clinical experimental study was developed in subjects whose ages were 18 to 45 yeas, attending the dental clinics of Santo Tomas University. They required operative dental treatments. The pain incidence in the two treatment groups and the possible secondary effects were registered.

Results: Forty one subjects were evaluated, $36.6 \%$ were in the acupuncture group $(\mathrm{n}=15)$ and $63.4 \%$ in the lydocaine group $(n=26)$. The distribution by gender showed that women $58.54 \%$ and men $41.46 \%$. The average of age was 25.7 and 31.15 years, respectively, and the range was between 18 and 45 years. Of the 41 subjects, $16(39.02 \%)$ felt pain during the treatment, 16 (39.02\%) had muscular drowsiness, 13 (31.71\%) felt pain when inserting the needle in the tissue, 8 (19.51\%) bled, 4 (9.76\%) presented irritation, $3(7.32 \%)$ registered headache, and $1(2.44 \%)$ was sweaty.

Conclusions: Both techniques were similar in effectiveness during the procedures of operative. By means of the acupuncture you can avoid $(60 \%)$ or diminish (40\%) the pain caused during the restorative procedures, eliminating for the patient the medication dose administered with this purpose.
\end{abstract}

Key words: Acupuncture, Lydocaine, Dental pain, Ancesthesia.

Recibido para publicación: 10 de agosto de 2004. Aceptado para publicación: 26 de octubre de 2004. 


\section{INTRODUCCIÓN}

Dentro de la profesión odontológica continuamente se trata de obtener analgesia, para evitar el dolor asociado con la consulta dental. El dolor es definido por la International Association for the Study of Pain (IASP) como una "Sensación desagradable y una experiencia emocional relacionada con el daño potencial o real de los tejidos». ${ }^{1}$

Algunas personas, con necesidades de tratamiento dental, son dificiles de tratar porque piensan que el odontólogo los va a lastimar con los procedimientos que utilice. A otras, el pensar que una de las opciones para evitar el dolor dental es la inyección de anestesia dentro de la cavidad bucal, les produce ansiedad y miedo.

En la actualidad existen diversos métodos de control del dolor (analgésicos, anestésicos, manejo psicológico, hipnosis, acupuntura, terapia neural, audio analgesia y aromaterapia, entre otros), que el profesional tiene a la mano para manejar esta sensación de los pacientes; sin embargo, el uso de algunos de éstos es muy limitado, posiblemente porque no se tienen los conocimientos pertinentes, lo que coloca a la anestesia local como el método más usado.

La acupuntura es una rama de la medicina tradicional china, que ha sido usada en Asia por más de 3000 años, como un método de tratamiento, que involucra la inserción de agujas en varios puntos del cuerpo con la intensión de curar enfermedades. ${ }^{36}$ Es una de las terapias alternativas más usadas en China. La literatura actual demuestra que da buenos resultados en diferentes tratamientos médicos en Occidente..$^{6-9}$ Además es una excelente alternativa para los pacientes alérgicos a los anestésicos locales, que presentan acidosis del tejido (absceso agudo o celulitis), o cardiopatías e hipertensión. ${ }^{329}$

En odontología son pocos los profesionales que la emplean; las publicaciones, aunque limitadas, muestran buenos resultados.

Por ello, el objetivo de este artículo es evaluar su efectividad, como anestesia en los procedimientos odontológicos de operatoria y comparárla con la anestesia local convencional con lidocaina

\section{MATERIALES Y MÉTODOS}

Se desarrolló un estudio experimental de tipo ensayo clinico, no controlado, con dos grupos de tratamiento (Acupuntura y Lidocaína).

La población de estudio estuvo conformada por 41 individuos de ambos géneros, en edades entre 18 y 45 años, durante Agosto de 2003, y Marzo de 2004.

La muestra fue seleccionada teniendo en cuenta las siguientes especificaciones de diseño: Confianza 95\%, P0der 95\%, Razón 1:1, diferencia promedio en el tiempo del efecto de la anestesia 1 minuto $01 \mathrm{~cm}$ en la intensidad de dolor después del procedimiento odontológico.

Se esperaba, al inicio del estudio, que una muestra de 26 individuos por grupo de tratamiento, para un total de 52 permitiera detectar diferencias estadísticamente significativas, entre la anestesia con acupuntura y la anestesia con lidocaína. Sin embargo, debido a la dificil aceptación del tratamiento con acupuntura por parte de los pacientes, se optó porque la presente investigación fuera la fase inicial de un proceso investigativo.

Se excluyeron del estudio individuos con dolor previo a la aplicación de anestesia, con compromiso sistémico, las mujeres embarazadas, los menores de edad, individuos con limitaciones cognitivas y estado emocional alterado.

Antes de la recolección de la información, se realizó una prueba piloto con el fin de estandarizar los procesos, definir los tiempos, evaluar los instrumentos y hacer los ajustes pertinentes.

Se obtuvo una historia médica detallada y un examen físico previo a la aplicación del tipo de anestesia para el procedimiento operatorio que pasara la unión amelodentinaria.

Después se explicaron los objetivos del estudio y el procedimiento, los pacientes leyeron, comprendieron, y firmaron un consentimiento informado, que fue aprobado por el Comité de Ética de la Universidad Santo Tomás. Previo al procedimiento operatorio que se realizó, los pacientes se asignaron por conveniencia a un grupo en el que recibieron anestesia con lidocaina y a otro grupo que recibió acupuntura. 
Se estudiaron variables sociodemográficas como género (masculino o femenino) y edad (escala ordinal dentro del rango de 18 a 45 años). El estado de salud general se determinó con base en la historia clínica del paciente.

Para la valoración del dolor se obtuvo la incidencia de dolor (presencia 0 ausencia) y la intensidad de dolor (Escala Análoga Visual - EAV).

Los efectos secundarios se establecieron mediante una escala nominal variable dicotómica (presencia o ausencia del efecto): Sangrado o hematoma, irritación, desmayo, adormecimiento muscular después del tratamiento, dolor de cabeza o sudoración.

\section{Análisis}

Los datos se analizaron aplicando medidas de tendencia central y de dispersión, y tablas de frecuencia, según la naturaleza y distribución de cada variable, en la totalidad de los individuos incluidos en el estudio. La base de datos se elaboró en Excel y el análisis en el programa STATA 8.0. ${ }^{30.31}$

Posteriormente se procedió a comparar los grupos mediante la prueba de $\mathrm{Chi}^{2}$ para variables nominales; y la $\mathrm{t}$ Student para la escala análoga visual y/o estadísticas no paramétricas, acordes con la distribución de la variable (prueba U de Mann - Whitney), considerando un nivel de significancia $\alpha=0.05$.

Se siguieron las recomendaciones éticas establecidas en la Resolución 008430 de 1993, del Ministerio de Salud de Colombia; según el título II, capítulo 1, en su artículo 11, clasifica este estudio de riesgo mayor que el mínimo.

\section{RESULTADOS}

\section{Descripción general}

En total se evaluaron 41 individuos. El 36.6\% pertenecían al grupo de acupuntura $(\mathrm{n}=15)$ y el $63.4 \%$ al grupo de lidocaina $(\mathrm{n}=26)$. La distribución por género mostró para mujeres $58.54 \%$ y para hombres $41.46 \%$, con un promedio de edad de 25.7 y 31.15 años respectivamente, dentro de un rango entre 18 y 45 años (Tabla 1).

\section{Valoración odontológica}

De los 41 individuos evaluados, 16 (39.02\%) sintieron dolor durante el tratamiento, 16 (39.02\%) tuvieron adormecimiento muscular, 13 (31.71\%) sintieron dolor al insertar la aguja en el tejido, 8 (19.51\%) presentaron sangrado 0 hematoma, 4 (9.76\%) presentaron irritación, $3(7.32 \%)$ registraron dolor de cabeza, y 1 (2.44\%) mostró sudoración.

\section{Análisis bivariado}

La sensación del dolor fue reportada por 10 (38.46\%) individuos del grupo de lidocaina y por 6 (40\%) individuos del grupo de acupuntura; el análisis comparativo muestra que no hay diferencia entre los tratamientos cuando se mide la variable en forma nominal dicotómica (ausencia o presencia de dolor) (Tabla 2, Figura 1).

La Prueba t de Student, para la intensidad de dolor registrado en la Escala Análoga Visual, mostró que no habia diferencia del nivel de dolor percibido por los individuos, que lo reportaron, en los dos grupos de tratamiento. Al observar que la variable de dolor, medida en Escala Análoga Visual (EAV), no registraba una distribución normal, se aplicó la prueba U de Mann - Whitney observando resultados similares $(P=0.5705)$ a los observados con la t Student, señalando que no hay diferencias entre la me-

\begin{tabular}{lccccc}
\hline \multicolumn{1}{c}{ VARIABLE } & \multicolumn{2}{c}{ Acupuntura $(n=15)$} & \multicolumn{2}{c}{ Lidocaina $(n=26)$} \\
\hline & \multicolumn{2}{c}{ Si } & $(\%)$ & $n$ & Si \\
Género masculino & 9 & 60,0 & 8 & 30,8 \\
Promedio de edad & 27,7 & -- & 23,75 & -- \\
Género femenino & 6 & 40,0 & 18 & 69,2 \\
Promedio de edad & 35,5 & -- & 26,8 & -- \\
\hline$n=41$ & & & &
\end{tabular}

Tabla 1. Variables sociodemográficas por grupo de tratamiento 


\begin{tabular}{|c|c|c|c|c|c|}
\hline \multirow[t]{3}{*}{ Variable } & \multicolumn{2}{|c|}{ Acupuntura ( $n=15$ ) } & \multicolumn{2}{|c|}{ Lidocaina ( $n=26$ ) } & \multirow[t]{2}{*}{$\mathbf{P}^{*}$} \\
\hline & & & & & \\
\hline & $n$ & (\%) & $n$ & (\%) & \\
\hline Dolor & 6 & 40,0 & 10 & 38,46 & 0.923 \\
\hline
\end{tabular}

$n=41$

* Test de $\mathrm{Chi}^{2}$

Tabla 2. Presencia de dolor sentido durante el procedimiento de operatoria

\begin{tabular}{|c|c|c|c|c|c|}
\hline \multirow[t]{3}{*}{ Efecto secundario } & \multicolumn{2}{|c|}{ Acupuntura ( $n=15$ ) } & \multicolumn{2}{|c|}{ Lidocaina $(n=26)$} & \multirow[t]{3}{*}{$\mathbf{P}^{*}$} \\
\hline & \multicolumn{2}{|c|}{$\mathrm{Si}$} & \multicolumn{2}{|c|}{$\mathrm{Si}$} & \\
\hline & $n$ & $(\%)$ & $n$ & $(\%)$ & \\
\hline Sangrado o hematoma & 7 & 46,7 & 1 & 3,8 & 0,001 \\
\hline Dolor al penetrar la aguja en el tejido & 5 & 33,3 & 8 & 30,8 & 8,865 \\
\hline Irritación & 2 & 13,3 & 2 & 7,7 & 0,558 \\
\hline Desmayo & 0 & 0,0 & 0 & 0,0 & $\cdots$ \\
\hline Adormecimiento muscular & 3 & 20,0 & 13 & 50,0 & 0,058 \\
\hline Dolor de cabeza & 0 & 0,0 & 2 & 11,5 & 0,172 \\
\hline Sudoración & 0 & 0,0 & 1 & 3,8 & 0,442 \\
\hline
\end{tabular}

$n=41$

* Test de Chi

Tabla 3. Presencia de efectos secundarios en cada uno de los grudos de tratamiento
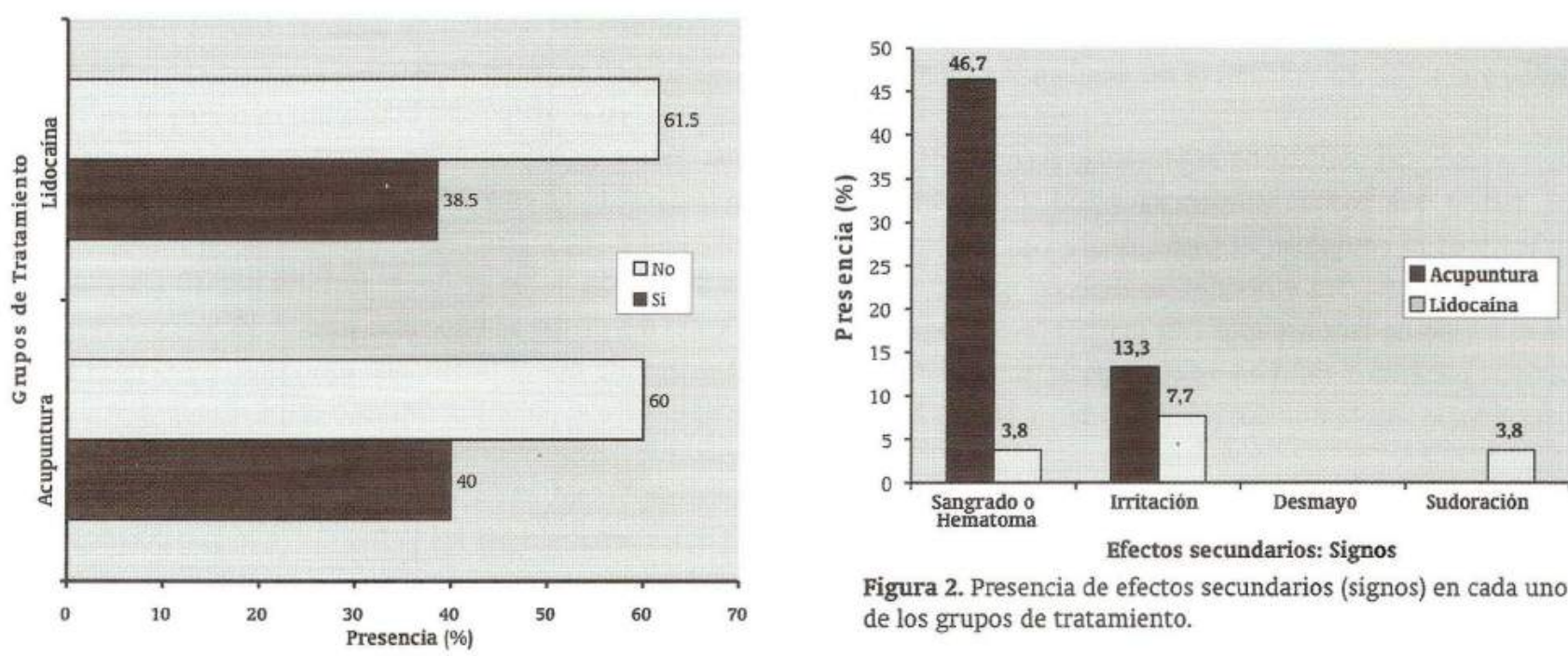

Figura 1. Presencia de dolor durante el procedimiento de operatoria.

Figura 2. Presencia de efectos secundarios (signos) en cada uno de los grupos de tratamiento.

89 


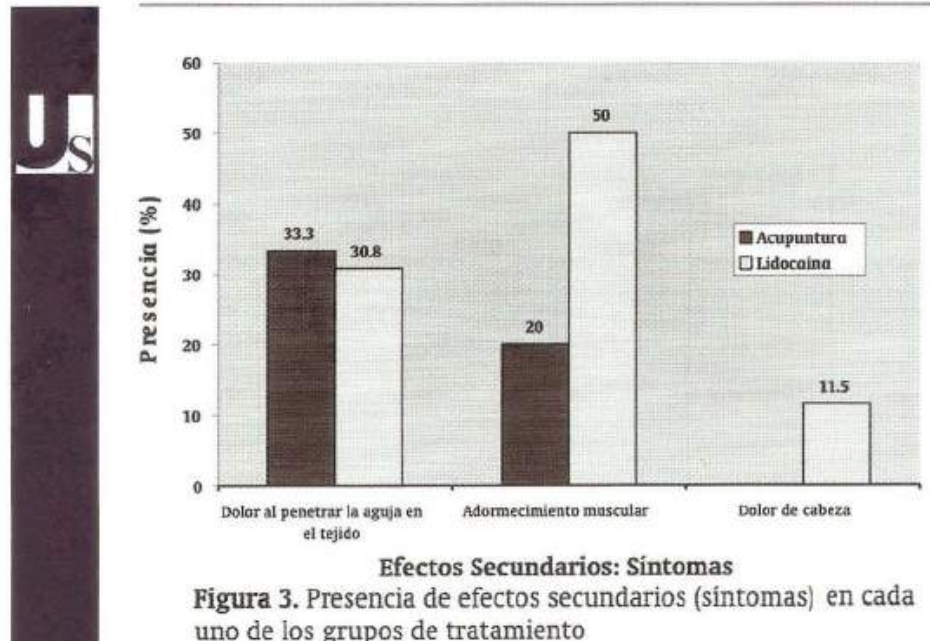
uno de los grupos de tratamiento

diana del dolor (según la EAV) del tratamiento con acupuntura, comparado con la mediana del dolor (según la EAV) del tratamiento con lidocaina.

Para el sangrado o hematoma, se encontró que hay diferencias estadísticamente significativas de presencia del efecto secundario en el tratamiento de acupuntura (46.7\%) comparado con el tratamiento con lidocaina (3.85\%) (Tabla 3).

$\mathrm{Al}$ analizar el dolor sentido por la inserción de la aguja en el tejido, la irritación y la sudoración, no se presentaron diferencias estadísticamente significativas entre ambos tratamientos (Tabla 3).

En el adormecimiento muscular, se halló una significancia marginal $(\mathrm{P}=0.058)$ entre el tratamiento con acupuntura (20\%) y con lidocaina (50\%) al ser analizado este efecto.

El resultado del análisis de la presencia de dolor de cabe$\mathrm{za}$, indica que no hay diferencias estadísticamente significativas al comparar el tratamiento con acupuntura y con lidocaina. Sin embargo, se destaca que mientras en el grupo de tratamiento con acupuntura ningún individuo presentó el síntoma, en el grupo de tratamiento con lidocaina, lo registraron dos (11.5\%) (Tabla 3, Figura 2).

\section{DISCUSIÓN}

La acupuntura es un método alternativo que permite brindar al paciente una serie de beneficios como la eliminación de la dosis farmacológica en los procedimientos odontológicos más comunes, como la operatoria dental. La presente investigación sugiere que la acupuntura es igualmente efectiva que la lidocaina, como anestésico local, en la prevención del dolor.

De los pacientes registrados en el grupo de acupuntura, 12 (80\%) prefirieron esta técnica anestésica a la convencional. Se observó un resultado similar en el artículo "Anestesia dental electrónica: estudio piloto». En éste se concluyó que de 20 individuos tratados con anestesia dental electrónica, 14 (70\%) indicaron que preferían este tipo de procedimiento a la inyección anestésica local. ${ }^{2}$

En cuanto a los efectos adversos, 13 (50\%) de los pacientes en el grupo de lidocaina presentaron adormecimiento muscular, comparado con $3(20 \%)$ de los registrados en el grupo de acupuntura, quienes reportaron que el adormecimiento era causado por tener la boca abierta y no por la técnica, como tal. Es de anotar que el paciente se mantenía inmóvil al ser anestesiado con acupuntura para evitar que la aguja se desplazara durante el procedimiento. En la literatura revisada se encontró que White y colaboradores referencian una incidencia de este síntoma del $0.3 \%{ }^{13}$

El sangrado o hematoma registrado en el grupo de acupuntura fue de $46.4 \%$; no obstante, para el registro de este signo se tuvo en cuenta hasta el más mínimo punto de sangre al retirar la aguja. Confrontado con el artículo de White y colaboradores, ${ }^{11}$ el evento más común fue el sangrado (incluyendo el hematoma) que tuvo un rango de incidencia entre 0 y $53 \%$ por practicante individual.

El resultado del análisis de dolor de cabeza destaca que mientras en el grupo de acupuntura ninguno presentó el sintoma, en el grupo con lidocaina, 2 (11.5\%) lo registraron. White y colaboradores, reportaron que en su estudio la incidencia de este sintoma fue de $0.1 \% .^{11}$

La sensación de dolor fue reportada por 10 (38.4\%) pacientes del grupo de lidocaína y por $6(40 \%)$ pacientes en el grupo de acupuntura; sin embargo, en el grupo de acupuntura el dolor fue definido como una molestia leve resistible. La intensidad de dolor registrado en la Escala Análoga Visual, mostró que no hay diferencia del nivel de dolor percibido por los individuos que lo reportaron en los dos grupos de tratamiento. Compatible con otros reportes de la literatura científica como los de Lao, en donde se encontró que la acupuntura daba más del doble de tiempo de anestesia postoperatoria, confrontada contra el uso de acupuntura placebo. ${ }^{12}$

90

Ustasalud Odontología 2004; 3: 86 - 91 
Este estudio puede ser la base para la realización de otros estudios en que la muestra sea mayor y los resultados puedan marcar significativamente las diferencias entre los dos procedimientos.

\section{BIBLIOGRAFÍA}

1. Internacional Association for the Study of Pain. Pain terminology [en línea] 1994 [fecha de acceso 20 de febrero de 2004]; URL disponible en: http://www.iasp-pain.org/terms-p.html\# Pain

2. Esposito CJ, Shay J, Morgan B. Anestesia dental electrónica: Estudio piloto. Quintessence Int (Ed. Esp) 1993; 24: 167 - 170.

3. Mejia S. Métodos físicos para el manejo del dolor: Acupuntura y sistemas afines. Bases para el Manejo del dolor en odontología. Colombia: Editorial Universidad de Antioquia; 1989.

4. Mayer DJ. Acupuncture: An evidence based review of the clinical literature. Annual Review of Medicine 2000; 51: 49.

5. Rosted P. Introduction to acupuncture in dentistry. British Dent J 2000; 189: $136-140$.

6. Chaitow L. Acupuntura y tratamiento del dolor. 3ra. edición. España: Ediciones Bellaterra; 1988.

7. Anonymous. Panel makes point about acupuncture. J Natl Cancer Inst 1997; 89: 1751.

8. Melchart D, Weidenhammer W, Streng A, Reytmair S, Hoppe A, Ernst E, Linde K. Prospective investigation of adverse effects of acupuncture in 97733 patients. Arch Intern Med 2004; 164: 104 - 105.

9. Domini P. Anestesia con acupuntura/analgesia para el control del dolor y la ansiedad en la práctica odontológica, Parte I: teoría y aplicación. En: Compendio de Clínica en Odontología 1994 - 1995; 1: 5 - 13

10. Domini P. Anestesia con acupuntura/analgesia para el control del dolor y la ansiedad en la práctica odontológica, Parte II: técnicas para su aplicación clínica. En: Compendio de Clínica en Odontología 1995 - 1996; 1: 4 - 13 .

11. White A, Hayhoe S, Hart A, Ernst E. Survey of adverse events following acupuncture (SAFA): A prospective study of 32000 consultations. Acupuncture in Medicine. 2001; 19: 84 - 92.

12. Lao L, Bergman S, Hamilton GR, Langenberg P, Berman B. Evaluation of acupuncture for pain control after oral surgery: A placebo controlled trial. Arch Otolaryngol Head Neck Surg 1999; 125: 567 - 572.

13. Lao L, Bergman S, Langenberg P, Wong RH, Berman B. Efficacy of Chinese acupuncture on postoperative oral surgery pain. Oral Surg Oral Med Oral Pathol Oral Radiol Endod. 1995; 79: $423-428$.

14. Gao D. Enciclopedia de la Medicina China. Primera Edición. 2000. Parramón Ediciones. España. Pág.: 7 - 207.
15. Türp J, Strub JR. La auriculopuntura y su aplicación en la estomatología (I), Introducción. Quintessence (ed. Esp). 1995; 8: 332 - 338.

16. Türp J, Strub JR. La auriculopuntura y su aplicación en la estomatología (II), Introducción. Quintessence (ed. Esp). 1995; 8: 383 - 385.

17. Lindall S. LIs acupuncture for pain relief in general practice costeffective? Acupuncture in medicine. 1999: 97-100.

18. Ezzo J. ¿Es eficaz la acupuntura para el tratamiento del dolor crónico? Una revisión sistemática. Rev Soc Esp Dolor. 2000; 8: 39 - 50.

19. Ernst E, White A. ¿Efectos adversos potencialmente letales de la acupuntura?, Una revisión sistemática sobre el tema. Rev Soc Esp Dolor. 1999; 6: 24 - 28

20. Fortinsky G. Complementary Dentistry: a more comprehensive term. J Can Dent Assoc. 2001; 67: 254 - 255.

21. Goldstein BH. Unconventional Dentistry: Part I. Introduction. J Can Dent Assoc. 2000; 66: 323 - 326.

22. Goldstein BH, Epstein JB. Unconventional Dentistry Part IV. Unconventional Dental Practices and Products. J Can Dent Assoc 2000; 66: 564 - 568

23. Goldstein BH. Unconventional Dentistry: Part V. Professional Issues, Concerns and Uses. J Can Dent Assoc. 2000; 66: 608-10.

24. Valdés F, Boch A. Acupuntura y electroacupuntura en el alivio del dolor de la osteoartrosis de la región lumbar. Rev Cubana Med Gen Integr. 2001; 17: $143-148$

25. Hammerschlarg R. Acupuncture: on what should its evidence base be based? Alternative Therapies in Health and Medicine. 2003; 9: 34

26. Anonymous. Analgesia. Acupuncture makes a point of pain relief. Nursing 2002; 32: 33.

27. Hitt E. Electro - acupuncture effective for anesthesia during in vitro fertilization. Medscape Today [on line] 2003. [fecha de acceso 13 de marzo de 2004]; URL disponible en: http://www.medscape.com/ viewarticle/463230

28. Posner GP, Sampson W. Chinese Acupuncture for heart surgery anesthesia. Sci Rev Alt Med 1999; 3:15 - 19

29. Beinfield H. Tribulations and trials: acupuncture study designs affect outcome. Altern Ther Health Med 2002; 8: 40-43. 30. Microsoft Excel 5.0, Microsoft Corporation 1997.

31. Stata Corporation 1999. Stata statistical software. Release 6.0. College Station TX: Stata Corporation. 